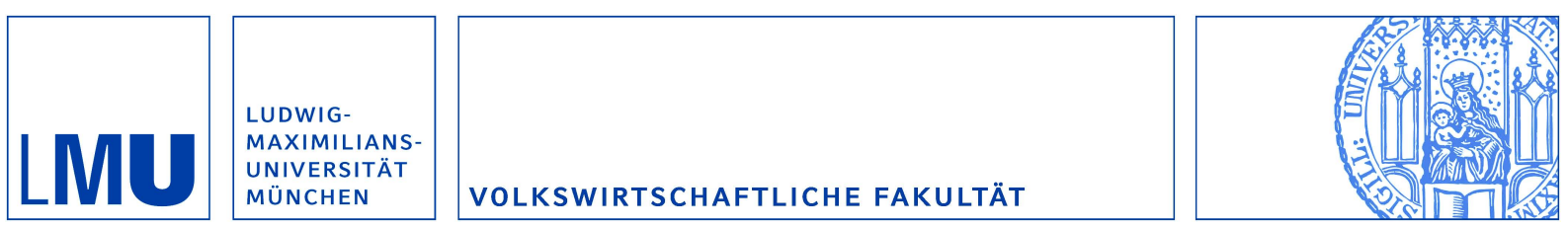

Mueller-Langer, Frank und Andreoli-Versbach, Patrick:

Leading-effect vs. Risk-taking in Dynamic Tournaments: Evidence from a Real-life Randomized Experiment

Munich Discussion Paper No. 2013-6

Department of Economics

University of Munich

Volkswirtschaftliche Fakultät

Ludwig-Maximilians-Universität München

Online at https://doi.org/10.5282/ubm/epub. 15452 


\title{
Leading-effect vs. Risk-taking in Dynamic Tournaments: Evidence from a Real-life Randomized Experiment
}

\author{
Frank Mueller-Langer* and Patrick Andreoli-Versbach** \\ * Max Planck Institute for Intellectual Property and Competition Law, \\ Munich Center for Innovation and Entrepreneurship Research (MCIER), \\ International Max Planck Research School for Competition and Innovation (IMPRS-CI) \\ ** Max Planck Institute for Intellectual Property and Competition Law, MCIER, IMPRS-CI, \\ Ludwig Maximilian University of Munich
}

June 17, 2013

\begin{abstract}
Two 'order effects' may emerge in dynamic tournaments with information feedback. First, participants adjust effort across stages, which could advantage the leading participant who faces a larger 'effective prize' after an initial victory (leading-effect). Second, participants lagging behind may increase risk at the final stage as they have 'nothing to lose' (risk-taking). We use a randomized natural experiment in professional two-game soccer tournaments where the treatment (order of a stage-specific advantage) and team characteristics, e.g. ability, are independent. We develop an identification strategy to test for leading-effects controlling for risk-taking. We find no evidence of leading-effects and negligible risk-taking effects.
\end{abstract}

Keywords: Tournaments, order effects, leading-effect, risk-taking, randomized natural experiments

JEL classification: C93; C21; D01; L83

Address: Dr. Frank Mueller-Langer (Corresponding author): Max Planck Institute for Intellectual Property and Competition Law, Marstallplatz 1, D-80539 Munich, Germany; E-mail: frank.mueller-langer@ip.mpg.de; Tel: +49 8924246 453. Patrick Andreoli-Versbach; E-mail: patrick.andreoli-versbach@imprs-ci.ip.mpg.de. 


\section{Introduction}

Tournaments are widely used in corporations, politics and sports to provide incentives to work hard or to select the best agents. A key aspect of tournaments is that participants are rewarded on the basis of their relative rather than absolute performance. In addition participants often compete in a dynamic setting with information feedback and under asymmetric conditions. Two fund managers acting on different markets and competing to attract new funds might get intermediate feedback of performance and change their strategy before the investors' choice of asset allocation. In the US the major party candidates are determined through a sequence of state-level primary elections where candidates can constantly monitor their interim rank and change their strategies accordingly. Most of the literature on tournaments points out how incentives mitigate the conflicting objectives between principal and agents inducing higher levels of effort. However, less is known on the effect of revealing information on relative performance during a dynamic tournament. In this setting effort is not the only choice variable and risk-taking might cause 'order effects'. By 'order effects', we mean the advantage or disadvantage to a player when performing either in a given sequence or under different conditions that are determined by the regulation of the tournament. Two distinct order effects might arise in a dynamic setting with intermediate information feedback where both effort and risk-taking are relevant. First, there might be a leading-effect. Teams taking the lead at the beginning of the tournament might experience an encouragement-effect and/or teams lagging behind might feel discouraged. This effect is due to the fact that the leading (lagging) player has an incentive to exert more (less) effort as she faces a larger (smaller) 'effective prize' from winning the second game (Konrad and Kovenock, 2009; Malueg and Yates, 2010). Second, teams lagging behind might increase risk-taking at final stages of the tournament as they have 'nothing to lose' (Cabral, 2003; Hvide, 2002).

In this paper we take advantage of a unique natural experiment with 1,146 observations where highly paid professionals have strong incentives to compete and know the setting very well. In two-game soccer knock-out competitions, teams are randomly drawn to have an advantage (home game) either in the first or second game. The team randomly drawn to play the first game at home wins the first game more often ( $53 \%$ home win, $26 \%$ draw and $21 \%$ away win) and thus might benefit from a leading-effect. The team playing the second game at home is more likely to lag behind after the first game and thus might increase risktaking in the second game. Using this real life situation that guarantees internal validity we investigate the selection efficiency of tournaments with information feedback and asymmetric initial conditions. 
The main concern of using a natural experiment as opposed to an experiment in the laboratory is that strategies, and in specific effort and risk choices, are unobserved. By exploiting our rich dataset we develop an identification strategy capable of distinguishing the relevance of each effect, i.e. leading-effect and risk-taking, on the winning probability of teams. Most of the literature focuses on how tournament design influences the behavior of participants and in particular on the incentive mechanism of tournaments (Knoeber and Thurman, 1994; Lazear and Rosen, 1981; Lynch, 2005). Another strand of literature elaborates on the comparison between tournaments and other performance schemes (Baker et al., 1994; Lazear, 2000; Oyer, 1998).

Less research is done on how the dynamic structure of tournaments affects the ex-ante winning probabilities of participants through order effects. This is of great importance for two reasons. First, from the perspective of the organizer, tournaments are often used as a selection mechanism to identify the best candidates, e.g. for job promotion or research grants. Better agents should win the tournament. This may not be the case when the regulation randomly attributes a considerable advantage to one player. Second, from the perspective of the participant, it is not fair if one player receives an advantage due to a randomized order of play. Thus, in dynamic tournaments, the order of interaction must be carefully designed. We benefit from a randomized natural experiment in soccer knock-out competitions with two games in which each team is randomly drawn to play either the first or the second game at home. Given the robustly identified result that home teams have an advantage (Clarke and Norman, 1995; Ferrall and Smith, 1999; Neave and Wolfson, 2003; Pollard, 1986), we investigate whether the random allocation of this advantage in either the first or second game has an impact on the probability of winning the knock-out. The setting of a knock-out allows us to go beyond what can usually be done in empirical work on selection efficiency in corporate tournaments where many variables are not observable and the data is not available. Sport is in many ways the perfect environment for testing economic theories about decision-making. ${ }^{1}$ There is an abundance of readily available data, the goals of participants in sporting contests are relatively uncomplicated and the outcomes are extremely clear. Szymanski (2003) concludes that sports data is a valuable source for economists trying to understand the relationship between tournament structure and effort choices and to test theoretical predictions against the data.

Our setting of a natural experiment allows us to study how the randomly assigned order of advantages might affect winning probabilities. Without order effects, teams' winning prob-

\footnotetext{
${ }^{1}$ Sports contests have been successfully used to show the use of mixed strategies (Chiappori et al., 2002), risk aversion (Pope and Schweitzer, 2011), cheating (Moul and Nye 2009), and the impact of the ability to adapt performance to pressure on overall career success (Dohmen, 2009; González-Díaz et al., 2012).
} 
abilities should be independent of whether they play the first or second game at home. We refer to this condition as 'neutral structure' of the tournament. If, however, order effects play a relevant role, professional and experienced teams in high stake environments will exploit such favorable conditions by changing their strategy and increase their winning probability. Our empirical analysis relates to two strands of literature on dynamic tournaments ${ }^{2}$ that focus on two aspects of order effects: leading-effect and risk-taking.

Regarding the leading-effect, Malueg and Yates (2010) and Ferrall and Smith (1999) study the existence of strategic effects in dynamic tournaments using individual and team level sports data, respectively. Malueg and Yates (2010) find that players in best-of-three tennis tournaments strategically adjust efforts across sets conditional on the intermediate score. Given equal ex-ante abilities, players that take the lead by winning the first set exert higher effort in the second set than the opponents as they face a larger 'effective prize' from winning the second set. ${ }^{3}$ Ferrall and Smith (1999) analyze data from team sports such as basketball, baseball and hockey and do not find evidence of leading-effects. ${ }^{4}$ Klumpp and Polborn (2006) model US presidential primaries that consist of a sequence of elections within a political party in different districts between two candidates. They find evidence of strategic effects. Consistent with empirical evidence, the winner of early districts is endogenously more likely to win later districts than the loser. A possible explanation for this mixed evidence is that incentives within teams may attenuate incentive effects across games which might explain why strategic effects are present in settings where individuals rather than teams compete (Ferrall and Smith, 1999).

In addition to effort choices, in many situations agents may also choose risk to influence their performance. Fund managers could pick riskier assets and managers opt for riskier investments or production technologies if they are lagging behind. In such cases the latter results on leading-effects do not hold true in general. When players can choose both effort and risk, players choose riskier actions and lower effort in equilibrium as compared to the equilibrium effort without risk-taking (Hvide, 2002). Also, when risk is the only choice variable, the selection efficiency of tournaments deteriorates (Hvide and Kristiansen, 2003). Cabral (2003) sets up a model in which firms can choose between a safe, low-variance and a risky,

\footnotetext{
${ }^{2}$ See Konrad (2009) for a thorough overview of the literature on dynamic contests.

${ }^{3}$ See also Konrad and Kovenock (2009) who analyze multi-battle, all-pay auctions and find that, with intermediate prizes, even a large lead by one player does not fully discourage the laggard. Without intermediate prizes, laggards may only drop out if they are lagging too far behind.

${ }^{4}$ Apesteguia and Palacios-Huerta (2010) study penalty kicks with randomly assigned order of who shoots first and find that teams randomly allocated to take the first kick win $60.5 \%$ of the shoot-outs. They ascribe this effect of sequential moves to 'psychological pressure' on the kicker of the second-kicking team. Note, however, that Kocher et al. (2012) cannot replicate this positive effect on teams kicking first in a larger sample of shoot-outs with 540 observations.
} 
high-variance research and development strategy. He provides sufficient conditions under which the firm lagging behind chooses a riskier strategy than the leader. As compared to a model without risk-taking, weaker players or players lagging behind choose riskier strategies because they have 'nothing to lose'.

Due to the difficulty to analyze effort and risk choices separately the empirical literature confirming the theoretical result of 'gambling for resurrection' is relatively underdeveloped. One notable exception are Genakos and Pagliero (2012) who study the impact of interim rank on risk-taking and performance in weightlifting competitions. They find that risk-taking takes an inverted-U relationship with interim rank where competitors that are ranked just behind the leader take more risk. In addition Chevalier and Ellison (1997) find that mutual funds with relatively low mid-year performance increase fund volatility, relative to the funds with relatively high mid-year performance.

The view that the player lagging behind increases risk-taking is not unanimous. Kräkel and Sliwka (2004) analyze a two-player tournament where players have asymmetric abilities. They show that depending on the interplay of effort and probability of winning and the degree of asymmetry between agents diverse equilibria are possible. For example, both agents may choose a high or low risk strategy. Nieken and Sliwka (2010) analyze a static model in which a leading player and a lagging player decide between risky and safe strategies. They show that the decisions depend on the correlation between contestants' outcomes of risky strategies. If the correlation is low, the player lagging behind increases risk whereas the leader plays safe in order to protect her lead. However, if the correlation is high, it might be optimal for the leader to follow the laggard's risky strategy. ${ }^{5}$ In a high-correlation environment it may well be attractive for the leading agent to imitate the competitor's risky strategy. Independent of whether the strategy fails or succeeds the relative position remains unchanged when the strategy can be exactly replicated. Thus, choosing the risky strategy becomes a means to protect the lead. If the outcomes of risky strategies are uncorrelated the imitation of risk-taking is ruled out from the outset.

Building on this theoretical framework on leading-effects and risk-taking we test two alternative predictions. First, teams playing the first game at home have an advantage at the beginning of the tournament and thus take the lead more often than teams playing the second game at home. This leading-effect might favor teams playing the first game at home by encouraging them to exert more effort or discourage the team lagging behind to exert

\footnotetext{
${ }^{5}$ Taylor (2003) sets up a model in which two heterogeneous fund managers in terms of midyear performance compete for new cash inflows at the end of the year. He shows that the outcome in which the lagging manager gambles and the leading manager indexes only holds if one of the managers is an exogenous benchmark. However, if both managers are active and the outcomes of their risky strategies are correlated, the leading manager is more likely to gamble.
} 
effort. ${ }^{6}$ Second, if the correlation between the contestants' outcomes of risky strategies is low, teams lagging behind at the end of the tournament might increase risk-taking as they have nothing to lose. This 'gambling for resurrection' could advantage the team playing the second game at home. If, however, the correlation is high teams might copy the rival's risk-taking strategy and risk-taking may be constant across teams and games. As both effects might be simultaneously taking place, we will first analyze risk-taking both dependent and independent on past performance. As the correlation between contestants' outcomes of risky strategies is rather high in soccer ${ }^{7}$ we show that risk choices are constant and do not depend on past performance. This result is in line with the theoretical predictions by Nieken and Sliwka (2010). We then test for leading-effects given constant risk choices and find no evidence of a significant effect.

The remainder of the paper is organized as follows: Section 2 describes the data. In Section 3 , we analyze risk-taking in each game of the knock-out and conditional on the result of the first game. Section 4 provides evidence on the absence of leading-effects. Section 5 discusses the results and Section 6 concludes.

\section{Data}

We construct a dataset with 1,146 games and thus 573 knock-outs where the home advantage is randomly assigned by the regulation of the Union of European Football Associations (UEFA). ${ }^{8}$ The data come from the UEFA. Table 1 summarizes the data. The dataset consists of games played in the UEFA Champions League and UEFA Europa League ${ }^{9}$ from 1955 until 2009. Observations from 564 games are from the period of 2005-2009 (80 from 2000-2004 and 502 from 1955-1999).

\section{[TABLE 1 HERE]}

\footnotetext{
${ }^{6}$ Note that both encouragement as well as discouragement-effect go in the same direction, i.e. they advantage the leading team. In this paper we therefore focus on measuring the impact of the random order of play on the selection efficiency of dynamic tournaments. The distinction between encouragement and discouragement-effect is beyond the scope of the paper.

${ }^{7}$ Grund and Gürtler (2005) analyze single soccer games. They show that as the opponent increases risktaking it is easier for the other team to score a goal. Intuitively, in the extreme risk-taking case where a team is lagging behind and the goalkeeper joins the striker in the last few minutes of the game to try to equalize the result, it will be very easy for the opposing team to score a goal.

${ }^{8}$ The UEFA is the administrative and controlling body of the European soccer association. UEFA represents most of the national soccer associations of Europe, runs national and club competitions and controls the prize money, regulations and media rights for those competitions.

${ }^{9}$ UEFA Champions League replaced the European Champion Clubs' Cup after season 1991/1992. UEFA Europa League replaced the UEFA Cup after season 2008/2009.
} 
For each game, we observe the date, result, location, knock-out round, tournament, and whether the team passed the knock-out round by goal difference, away goals rule, extra time, or penalty kicks. Table 2 summarizes the relative importance of each UEFA regulation. ${ }^{10}$ The goal difference rule is by far the most important one. In the last column, we summarize the winning probability of a single game from the perspective of the home team. The data on home winning probabilities show that the place where the game is disputed significantly affects the outcome. This result is therefore in line with previous literature on the advantage of playing at home. ${ }^{11}$

\section{[TABLE 2 HERE]}

\section{$3 \quad$ Natural experiment}

We study a randomized natural experiment in which the order of an advantage, and thus treatment and control group, are determined via explicit randomization. One team is drawn to play the first game home, and the other to play the second game home. In this natural experiment professionals know exactly the tournament's setting, the payoffs and incentives are very high, and the process of allocating teams is random. Page and Page (2007) analyze the same knock-out setting. The most important difference between this paper and Page and Page (2007) is that we develop an identification strategy capable of distinguishing between the leading-effect and risk-taking. In addition Page and Page (2007) do not distinguish between random and non-random knock-out rounds. The key difference is that in the non-random knock-out rounds the regulation states that better teams play their last game home. Page and Page (2007) find a positive and significant effect of playing the second game at home ( $55 \%$ vs. $45 \%$ ), but cannot distinguish between the risk-taking effect and the higher ability of teams playing the last game home. These two confounding variables would favor the teams playing the second game at home, but it is not clear which one, if any, should be the driver of the $45 \%$ vs. $55 \%$ advantage.

In contrast, we focus on knock-out rounds where the order of the advantage is randomly assigned. This allows us to exploit the properties of a natural experiment and specifically

\footnotetext{
${ }^{10}$ See the appendix for the structure of the knock-out and the four ways of winning the knock-out.

${ }^{11}$ The average goal difference between home and away team is .72 goals in our sample of 1,146 games. This positive difference is significantly different from zero (same number of home and away goals) at a $p$-value lower than .01. Other studies (Carmichael and Thomas, 2005; Greenhough et al., 2002; Clarke and Norman, 1995; Pollard, 1986) estimate this advantage to be between .43 and .66 goals in national soccer leagues. However, they also find that the home advantage increases significantly in the geographical distance between the two teams. Hence, our findings which are based on international games between more distant teams are in line with the existing literature on the home advantage.
} 
the fact that team characteristics as ability and the treatment effect, i.e. the order of home games, are independent. ${ }^{12}$

In the final phase of the major European soccer tournaments, such as the Champions League and the Europa League, teams are randomly drawn to play against each other with a time interval of one to three weeks between the two games. There is a fundamental difference between knock-outs in the final rounds of the tournament, i.e. the quarter- and semi-finals, and the qualification rounds for the main tournament. For instance, Article 8.07 of the Regulations of the UEFA Champions League 2008/09 prescribes that "the ties are determined by means of a draw. The club drawn first plays the first leg of the tie at home". With respect to the qualification phase, Article 8.01 of the Regulations of the UEFA Champions League 2008/09 states that "the UEFA administration seeds clubs for the qualifying phase, the play-offs and the group stage (...) in accordance with the club coefficient ranking established at the beginning of the season (...)". ${ }^{13}$ Thus, teams are not randomly drawn to play in a given order, but better teams are allocated to play the second game at home. In light of this fundamental difference, causal inference about the order can only be drawn from the final phase. Therefore, we analyze the 1,146 games where the home advantage is randomly assigned.

Because of the random draw, the Average Treatment Effect (ATE) is defined as the difference between the two groups' mean winning probabilities. Let $y_{S}\left(y_{F}\right)$ denote the winning probability of a team playing the second (first) game at home, and let $w$ denote the allocation of home and away games. The average treatment effect is $A T E_{S}=A T E_{F}=E(y \mid w=$ $1)-E(y \mid w=0)$, if $w$ is statistically independent of $y_{S}$ and $y_{F}$.

As the theoretical work on dynamic tournaments points out, two effects going in opposite directions may emerge from such a setting. First, teams may get discouraged (encouraged) after an initial loss (victory) which gives them information feedback on their opponent's ability. Second, teams lagging behind might choose riskier strategies. ${ }^{14}$

The econometric problem arising from these different strategies can be illustrated as follows. Let $\varepsilon_{S, t}\left(\varepsilon_{F, t}\right)$ be the risk-taking in the $t=$ first, second game of team $S(F)$ that plays the

\footnotetext{
${ }^{12}$ We also tried an identification strategy similar to Malueg and Yates (2010) and selected equally skilled teams using the smallest possible positive goal difference of the first game, i.e. a one goal lead by the home team. The results on leading-effect and risk-taking were unchanged.

${ }^{13}$ Article 6.09 states that "the quarter-final pairings are determined by means of a draw. The quarter-finals are played under the cup (knock-out) system, on a home-and-away basis (two legs)." Article 6.10 prescribes that "the semi-final pairings are determined by means of a draw." The same rules apply to the Round of last 16 as well as the quarter- and semi-finals of the Europa League.

${ }^{14} \mathrm{~A}$ natural way in which coaches of teams that are lagging behind might increase risk-taking is by substituting defensive players by more offensive ones. Using data on the German soccer league Grund and Gürtler (2005) provide evidence that coaches adopt this strategy. However, risk-taking does not pay off in this setting.
} 
second (first) game at home. Then $E(y \mid w=\{1,0\}) \neq E\left(y \mid w=\{1,0\}, \varepsilon_{F, t}, \varepsilon_{S, t}\right)$, as both choice variables $\varepsilon_{F, t}$ and $\varepsilon_{S, t}$ are correlated with the random allocation of the advantage. If we do not control for them, we would have a biased estimator of order effects as the analysis would suffer from an omitted variable bias. Therefore, we must first understand what type of model drives teams' risk choices. As shown by Nieken and Sliwka (2010), if the correlation between contestants' outcomes of risky strategies is low, then there is an incentive for the team lagging behind to increase risk. If this correlation is high, teams tend to copy the rival's strategy more often. Specifically to our setting, if the correlation is high $F$ anticipates the risk strategy that $S$ chooses in the second game and chooses the same risk level in the first game. Under this hypothesis $E(y \mid w=\{1,0\})=E\left(y \mid w=\{1,0\}, \varepsilon_{F}, \varepsilon_{S}\right)$ as risk choices are identical across games of the knock-out and thus the $A T E$ is the difference of the winning probabilities given the order of the home game. In the next section we provide indirect empirical evidence that the correlation between contestants' outcomes of risky strategies is rather high and thus risk-taking is indeed constant across games and unconditional on past performance.

\subsection{Risk-taking unconditional on past performance}

The effects of risk-taking can be tested in two ways: first, by comparing the distribution of results (home win, draw, away win) in the first and second game, and second, by comparing the number of goals scored across games. These two measures are interrelated and capture whether there is evidence that risk-taking influences the results across games. Intuitively, if risk-taking differs across games, it should shift the distribution of results towards the extremes (more home/away wins) and increase the number of goals at the end of the knockout, when one team is lagging behind. We start by analyzing the first measure, i.e. the distribution of results (home (win), draw and away (win)), in the two games.

\section{[FIGURE 1 HERE]}

If risk-taking plays an important role at the end of the knock-out, we should observe significantly fewer draws in the second game and more home/away wins as the effect of an increase in risk would be to shift probability from the median (draw) to the extremes (win/lose). Teams lagging behind towards the end of the knock-out have nothing to lose and thus might be indifferent between a draw and a defeat. Note that monetary incentives to win the knock-out are very high and often exceed $€ 3$ million, ${ }^{15}$ which points to the fact that winning the knock-out really matters. Figure 1 shows the relative frequency of each outcome

\footnotetext{
${ }^{15}$ For instance, in addition to the revenues generated from broadcasting, merchandising, sponsoring and tickets, each of the 32 teams that play in the Champions League receives a minimum payment of $€ 9.3$
} 
for 1,146 games where the home advantage is randomly assigned. To test the hypothesis of constant risk-taking in both games we perform a non-parametric Kolmogorov-Smirnov test on the distribution of outcomes. The test cannot reject equality of distribution across games with a $p$-value of .89 . The distributions do not differ, a finding that is in line with our hypothesis that risk choices are constant over games of the knock-out. The second measure we use to evaluate the relevance of risk-taking is the distribution of the sum of the home and away team goals across games. If at the end of the knock-out one team is lagging behind and has an incentive to increase risk-taking, we should observe that the distribution of the sum of the goals is more skewed to the right (more goals) in the second game. The average number of goals in the first (second) game is 2.48 (2.7) implying a difference of .22 goals across games. Doing a two-sided $t$-test with 573 observations per sample, the .22 difference is statistically significant at a $p$-value of .031. While this might point to an increase in risktaking at the end of the knock-out, the magnitude is very small, one goal more every five games, which points to a negligible effect of risk-taking on the outcome of the knock-out.

\section{[FIGURE 2 HERE]}

To confirm this assertion we plot the histogram of the sum of home and away team goals in the two games in Figure 2 and perform a two-sample Kolmogorov-Smirnov test to evaluate whether the distributions are significantly different. Using our 1,146 games, 573 in the first game and 573 in the second game, we cannot reject equality of distribution functions at a $p$-value of .24. Thus, while there is some evidence that the number of goals increases in the second game, the magnitude is very small and the distribution of the sum of goals is not statistically different across games.

\subsection{Risk-taking conditional on past performance}

Even though we cannot reject that the distributions of goals are equal across games, strategies across games might not be independent and thus teams might react to past performance. In particular, teams that lost the first game might increase their risk-taking. This strategy, if effective, might lower the selection efficiency of tournaments as predicted by Hvide and Kristiansen (2003). We test this by relating the sum of home and away goals in the second game to the goal difference (home-away) in the first game. If risk-taking depends on past performance we would expect that the sum of goals in the second game increases if the absolute goal difference in the first game increases, i.e. we should observe a U-shaped relation

million plus rewards of reaching the round of last 16 ( $€ 3$ million), the quarter-finals ( $€ 3.3$ million), the semi-finals ( $€ 4.2$ million) and the final (€ 5.6 million). UEFA (2012). Financial Report 2010/11. UEFA, Nyon: Switzerland. 
between past performance (goal difference) and the sum of goals. ${ }^{16}$ A team lagging behind in the second game, i.e. with a negative goal difference in the first game, might increase risktaking as they have nothing to lose which should increase the number of goals in the second game. The team lagging behind might substitute a defensive player for a more offensive one as shown in Grund and Gürtler (2005) which increases the likelihood that the team lagging behind equalizes while at the same time makes it easier for the leading team to score. Both effects point to an increase of the sum of home and away goals in the second game.

If, however, the correlation between contestants' outcomes of risky strategies is relatively high, it might be optimal for both the leading and lagging team to choose the same risk level and thus the sum of goals in the second game should be independent from the goal difference of the first game.

In Figure 3 we plot the mean, interquartile range and $95 \%$ confidence interval of the sum of home and away goals in the second game conditional on the goal difference (home-away) in the first game. We can test two alternative hypotheses regarding risk-taking across the two games. First, teams lagging behind increase their risk-taking. Thus there should be a positive relation between the number of goals that a team is lagging behind after the first game and the total number of goals scored in the second game. Second, risk-taking is constant across games and thus there is no relation between past goal difference and the sum of goals in the second game. As Figure 3 shows, the sum of goals in the second game is independent of the goal difference in the first game. In order to test the graphic relation we perform an OLS regression with robust standard errors as given by

$$
\text { sumgoals }_{H+A, 2}=\beta_{0}+\beta_{1} \text { goaldif }_{H-A, 1}+\beta_{2} \text { goaldif }_{H-A, 1}^{2}+u_{2}
$$

We define sumgoals sutA,2 $_{H}$ as the sum of home $(H)$ and away $(A)$ goals in the second game. $u_{2}$ is the error term. Let goaldif $f_{H-A, 1}\left(\right.$ goaldi $\left._{H-A, 1}^{2}\right)$ be the difference (squared) between home and away goals in the first game. If risk-taking plays a role we should observe a Ushaped relation between past performance (goal difference) and the sum of goals. Thus, $\widehat{\beta}_{1}$ should be insignificant and $\widehat{\beta}_{2}$ should be positive and significant. Estimating the regression using OLS we find that the constant is positive, 2.69, and significant at the $1 \%$ level. $\widehat{\beta}_{1}$ and $\widehat{\beta}_{2}$ are highly insignificant with a $p$-value of .77 and .97 , respectively. These results confirm the graphic evidence that there is no relation between past performance and current risk-taking.

\footnotetext{
${ }^{16}$ If $S$ is lagging behind by a large goal difference after the first game it could increase risk-taking which in turn leads to a higher total number of goals in the second game. Symmetrically, if $F$ is leading by a large number of goals it will be easier for $F$ to score additional goals in the second game as $S$ increases its risk-taking. In contrast, if the goal difference between teams is rather low, we would expect risk-taking and thus the total number of goals in the second game to be low.
} 
[FIGURE 3 HERE]

\section{On the absence of leading-effects}

We can test for leading-effects using a natural experiment in soccer knock-outs if risk-taking is constant across games and teams. As we show in the previous section, there is no evidence that risk-taking plays a significant role. Thus, we can exploit the properties of the natural experiment.

The two-game structure in soccer competitions should be a neutral structure absent of order effects. The overall winning probability for $S$, the team playing second home, is $51.8 \%$. While the point estimate is slightly above $50 \%$, it is far from being significantly so. For preliminary evidence we perform a two-sided binomial test with the hypothesis that the order of play does not significantly influence the probability of winning. Performing the test with a sample size of 1,146 games and $H_{0}: 50 \%$, we get a $p$-value of .31 so that we cannot reject the hypothesis of the mean to be $50 \%$.

\section{[TABLE 3 HERE]}

In Table 3, we perform an in-depth analysis by adding various control variables. We use a logit model ${ }^{17}$ where the dependent variable, win $_{i}$, is binary and equals 1 if the team $i$ wins the knock-out. As our observations are not independently drawn from the same population, but one team winning the knock-out implies the other losing it, we cluster the standard errors by the knock-out ID.

In the first specification of Table 3, we regress $w_{i n}$ on $S H_{i}$, a dummy indicating whether team $i$ plays the second game at home. As specification 1 shows, $S H$ is indeed insignificant. In the second specification, we add an interaction term between $S H$ and time dummy variables from 1955 to 2009 to test whether time fixed effects are present. In specification 3, we add an interaction between Champions League games and $S H$. As better teams play for higher stakes in this competition as opposed to the Europa League, it might be that these teams are more capable of exploiting their advantage deriving from order effects. As the regression results show, this is not the case. Statistically, there is no significant difference between $\mathrm{SH}$ in Champions League or Europa League. In specification 4, we include both time fixed effects and the Champions League dummy but results are unchanged. In specification 5, we include round dummies. As it might be that order effects are stronger in the final knock-out rounds of the tournaments when stakes are highest, we include four interactions between round dummies and $S H$. As specification 5 shows, none of them is individually significant

\footnotetext{
${ }^{17}$ Using a probit model instead does not qualitatively change the results.
} 
at the usual confidence levels. In the last specification we add all control variables but none has a significant effect on the probability of winning given that the team played the second game home and $S H$ is insignificant.

In this two-game tournament context, we find no evidence for leading-effects. On average, teams playing home second have a slight advantage, but this is not significantly different from no-advantage. The evidence provided in Table 3 and in the previous section on risk-taking shows that allocating symmetric advantages at different stages of tournaments is fair from the players' perspectives and guarantees selection efficiency from the tournament organizer's perspective.

\section{Discussion}

Our empirical strategy rests on two testable predictions from the theoretical literature on tournaments (leading-effect and risk-taking). Even though our strategy addresses each prediction separately there might be some concerns on the strategies and beliefs teams have during the game which we do not observe. For example, we do not observe the beliefs of players or coaches. If they believe to be disadvantaged by their order of home play, this might cause an (unobservable) decrease in effort. While such beliefs are unobservable, we address the two main causes of order effects and provide evidence that neither of them plays a significant role. In soccer there might be additional unobserved strategies, as for example players might get substituted for the risk of injuries or the coach might change formation. While it is unquestionable that such decisions may play a role, the advantage of having a natural experiment is that the teams' characteristics are uncorrelated with the treatment effect. Thus, additional strategic behavior should be uncorrelated with the treatment effect, i.e. the order of the advantage, as we control for the two main potential causes of order effects. Furthermore, in other sports like National Basketball Association (NBA) basketball, teams might be more likely to intentionally lose games at the end of the regular season because of the incentives they face (Taylor and Trogdon, 2002). This is not a concern in our setting, where the seedings for the tournament depend on the end-of-year rankings in the national leagues and (financial) incentives to compete in every game of the tournaments are very high. ${ }^{18}$ An additional concern might be that our setting is not perfectly symmetric. Even though $93 \%$ of the knock-outs finish after the second game, a minority continues to supplementary times (4\%) and eventually finishes after the penalty kicks (3\%), as is shown in Table 2. In knock-outs where the competition is tight, $S$ might adopt a strategy which increases the probability of reaching the extra time. Then $S$ will be playing the extra time

\footnotetext{
${ }^{18}$ See footnote 15.
} 
at home, where it has an advantage. ${ }^{19}$ While these issues might be a concern, players are highly paid professionals who exactly know the setting of the game and have high incentives to pass the knock-out round. If they could get advantaged by their order of home play, they would exploit this advantage. At optimum, coaches and players maximize their winning probability given the random order of home play.

\section{Conclusion}

Tournaments are widely used for two main purposes: as a selection mechanism and to provide incentives to work hard. While many tournaments are dynamic, little is known about the effect of revealing information during the tournament on participants' effort and risk choices. We focus on the selection efficiency of dynamic tournaments. If the structure of multi-game tournaments systematically distorts winning probabilities because of leading-effects or risktaking, tournaments may not be an efficient selection mechanism when multiple repetition under different conditions is needed. Some players could benefit from an advantaged position and the winner may not be the best participant, but the luckiest one. In this paper, we analyze the presence of order effects in dynamic tournaments with asymmetric conditions. We define order effects as whether the random order of a temporary advantage for a team in multi-game tournaments has an impact on its probability of winning. From a theoretical perspective two alternative and opposing hypotheses have been proposed. First, there might be a leading-effect. The winner of the first game faces a higher 'effective prize' from winning the second game than the first-game loser. This encourages the leading team and/or discourages the team lagging behind to exert effort. Second, agents lagging behind might increase risk-taking as they have nothing to lose.

Using a natural experiment with 1,146 observations in professional sports competitions where highly paid professionals play in tournaments with strong (financial) incentives, we develop an empirical strategy to distinguish between the two order effects highlighted by theoretical work on tournaments: leading-effect and risk-taking. In two-game soccer knock-out competitions, teams are randomly drawn to play either the first or second game at home, and thus have an advantage either early in the knock-out round or later. Before analyzing the conditional winning probability on the randomly assigned order of the advantage we provide evidence that risk-taking is constant across games and does not increase in response to negative past performance. Our empirical evidence suggests that players anticipate the opponent's risk-taking across games and adapt their risk-taking behaviour accordingly. The

\footnotetext{
${ }^{19}$ We performed the leading-effect analysis on different sub-samples depending on when the knock-out ended (regular time, extra time and penalty kicks), but results were unchanged.
} 
team playing the first game at home, $F$, anticipates that the opponent might lag behind and increase risk-taking in the second game. Consequently, $F$ increases risk-taking in the first game as well, and risk choices are constant across games.

As we do not find any evidence of risk-taking, we perform a series of regressions relating the winning probability to the order of the advantage and other covariates. We find that teams have statistically the same winning probability irrespective of whether they have an advantage in the first or second game. Using a unique and large dataset with 1,146 games where advantages are randomly assigned, we show that teams playing first (second) home win $48.2 \%(51.8 \%)$ of the knock-outs, not statistically different from $50 \%$ with a $p$-value of .31. In the regression analysis we confirm this finding using a logistic regression model with clustered standard errors at knock-out-ID. In addition, we add other control variables as time fixed effects, type of tournament and knock-out round dummy variables. The results are unchanged. In all specifications, the order of the advantage is never significant.

Our findings using team sport data are consistent with Ferrall and Smith (1999). In contrast, papers using data on individuals as Malueg and Yates (2010) and Genakos and Pagliero (2012) find evidence of strategic effects. A possible explanation for this difference between teams and individuals is provided by Ferrall and Smith (1999) who argue that incentives within teams may attenuate incentives between teams. Our results suggest that if the setting is known by participants and individuals are competing in teams the timing of symmetric advantages seems irrelevant. This guarantees selection efficiency and fairness for participants.

Acknowledgement: Both authors have contributed equally to this paper and the alphabetical order in which the authors appear is randomly drawn. We wish to thank Tim Barmby, Charles Knoeber, Dan Kovenock, Uwe Sunde, Theofanis Tsoulouhas, and the participants of the "Tournaments, Contests and Relative Performance Evaluation" conference at NC State (Raleigh, NC, USA). We are also very grateful to Luis Cabral, Luis Garicano, Christos Genakos, Hans Hvide, Martin Kocher, Kai Konrad, Tobias Kretschmer, Sandra Ludwig, Florian Morath, Lionel Page, Ignacio Palacios-Huerta, Klaus Schmidt, Monika Schnitzer, Joachim Winter, and seminar participants at the Ludwig Maximilian University of Munich and the Max Planck Institute for Tax Law and Public Finance for valuable comments. We gratefully acknowledge financial support from the Deutsche Forschungsgemeinschaft through GRK 801. 


\section{Appendix}

There are four ways of winning the knock-out, which apply in the following order. First, the goal difference rule states that the team that scores more goals on aggregate in the two games qualifies for the next knock-out round. Second, the away goals rule prescribes that if the two teams score the same number of goals over the two games, the team that scores more away goals qualifies for the next knock-out round. Third, Article 7 of the Regulations of the UEFA Champions League 2008/09 states that "if both teams score the same number of goals at home and away, two 15-minute periods of extra time are played at the end of the second leg". Fourth, "if no goals are scored during extra time, kicks from the penalty mark (...) determine which club qualifies for the next stage."

The knock-out competition is structured in six steps.

1) Teams $F$ and $S$ are randomly allocated to play either the first or the second game at home.

2) The first game is played. Assume without loss of generality that $F$ is the home team in the first game.

3) The second game is played ( $S$ is the home team now).

4) If the sum of the goals of $S$ is strictly larger (smaller) than those of $F$, the game ends and $S(F)$ wins the knock-out. If the sums are equal, the team that scored more away goals wins. If also the away goals are equal the game continues at $S$ 's venue.

5) Supplementary time is played. The team that scores more goals in the supplementary time wins.

6) If both teams score the same number of goals, penalty kicks are used to determine the winner.

\section{[FIGURE 4 HERE]}

The continuation of the game at $S$ 's venue could be the explanation for the higher point estimate of teams playing the second game home, $51.8 \%$. As we show in Table 3 , there is no significant advantage from playing second, but the fact that the game is played on the field of the team playing home second is certainly a small advantage. The percentage of games ending after the second game for the entire dataset with 1,146 observations is $93 \%$, while $4 \%$ of the games end after supplementary times and $3 \%$ after penalty kicks. The probability of winning for $S$ conditional on reaching supplementary time (penalty kicks) is $57 \%$ (55\%), not significantly different from $50 \%$. 


\section{References}

Apesteguia, J., Palacios-Huerta, I., 2010. Psychological pressure in competitive environments: Evidence from a randomized natural experiment. American Economic Review 100(5), $2548-2564$.

Baker, G. P., Gibbs, M., Holmstrom, B., 1994. The wage policy of a firm. Quarterly Journal of Economics 109, 921-955.

Cabral, L. M. B., 2003. R\&D competition when firms choose variance. Journal of Economics and Management Strategy 12(1), 139-150.

Carmichael, F., Thomas, D., 2005. Home-field effect and team performance: Evidence from English premiership football. Journal of Sports Economics 6(3), 264-281.

Chevalier, J., Ellison, G., 1997. Risk taking by mutual funds as a response to incentives. Journal of Political Economy 105, 1167-1200.

Chiappori, P.-A., Levitt, S., Groseclose, T., 2002. Testing mixed-strategy equilibria when players are heterogeneous: The case of penalty kicks in soccer. American Economic Review 92(4), 1138-1151.

Clarke, S. R., Norman, J. M., 1995. Home ground advantage of individual clubs in English soccer. Journal of the Royal Statistical Society: Series D 44(4), 509-521.

Dohmen, T. J., 2008. Do professionals choke under pressure?. Journal of Economic Behavior and Organization 65 (3-4), 636-653.

Ferrall, C., Smith, A. J., 1999. A sequential game model of sports championship series: Theory and estimation. Review of Economics and Statistics 81(4), 704-719.

Genakos, C., Pagliero, M., 2012. Interim rank, risk taking and performance in dynamic tournaments. Journal of Political Economy 120(4), 782-813.

González-Díaz, J., Gossner, O., Rogers, B. W., 2012. Performing best when it matters most: Evidence from professional tennis. Journal of Economic Behavior and Organization 84(3), 767-781. 
Greenhough J., Birch, P. C., Chapman, S. C., Rowlands, G., 2002. Football goal distributions and extremal statistics. Physica A: Statistical Mechanics and its Applications 316(1), 615-624.

Grund, C., Gürtler, O., 2005. An empirical study on risk-taking in tournaments. Applied Economics Letters 12(8), 457-461.

Hvide, H. K., 2002. Tournament rewards and risk taking. Journal of Labor Economics 20(4), 877-898.

Hvide, H. K., Kristiansen, E., 2003. Risk taking in selection contests. Games and Economic Behavior 42, 172-181.

Klumpp, T., Polborn, M., 2006. Primaries and the New Hampshire Effect. Journal of Public Economics 90, 1073-1114.

Knoeber, C. R., Thurman, W. N., 1994. Testing the theory of tournaments: An empirical analysis of broiler production. Journal of Labor Economics 12, 155-179.

Kocher, M., Lenz, M., Sutter, M., 2012. Psychological pressure in competitive environments: New evidence from randomized natural experiments. Management Science 58(8), 1585-1591.

Konrad, K. A., 2009. Strategy and Dynamics in Contests. New York: Oxford University Press.

Konrad, K. A., Kovenok, D., 2009. Multi-battle contests. Games and Economic Behavior $66,256-274$.

Kräkel, M., Sliwka, D., 2004. Risk taking in asymmetric tournaments. German Economic Review 5(1), 103-116.

Lazear, E. P., 2000. The power of incentives. American Economic Review 90(2), 410-414.

Lazear, E. P., Rosen, S., 1981. Rank-order tournaments as optimum labor contracts. Journal of Political Economy 89(5), 841-864.

Lynch, J. G., 2005. The effort effects of prizes in the second half of tournaments. Journal of 
Economic Behavior and Organization 57(1), 115-129.

Malueg, D. A., Yates, A. J., 2010. Testing contest theory: Evidence from best-of-three tennis matches. Review of Economics and Statistics 92(3), 689-692.

Moul, C. C., Nye, J. V.C., 2009. Did the Soviets collude? A statistical analysis of championship chess 1940-1978. Journal of Economic Behavior and Organization 70(1/2), 10-21.

Neave, N., Wolfson, S., 2003. Testosterone, territoriality, and the 'home advantage'. Physiology and Behavior 78(2), 269-275.

Nieken, P., Sliwka, D., 2010. Risk-taking tournaments: Theory and experimental evidence. Journal of Economic Psychology 31(3), 254-268.

Oyer, P., 1998. Fiscal year ends and nonlinear incentive contracts: The effect on business seasonality. Quarterly Journal of Economics 113(1), 149-185.

Page, L., Page, K., 2007. The second leg home advantage: Evidence from European football cup competitions. Journal of Sports Sciences 25(14), 1547-1556.

Pollard, R., 1986. Home advantage in soccer: A retrospective analysis. Journal of Sports Sciences 4(3), 237-248.

Pope, D. G., Schweitzer, M. E., 2011. Is Tiger Woods loss averse? Persistent bias in the face of experience, competition, and high stakes. American Economic Review 101(1), 129-157.

Szymanski, S., 2003. The economic design of sporting contests. Journal of Economic Literature 41(4), 1137-1187.

Taylor, B. A., Trogdon, J. G., 2002. Losing to win: Tournament incentives in the National Basketball Association. Journal of Labor Economics. 20(1), 23-41.

Taylor, J., 2003. Risk-taking behavior in mutual fund tournaments. Journal of Economic Behavior and Organization 50(3), 373-383. 


\section{$9 \quad$ Tables and Figures}

Table 1: Summary of the data

Competition

Phase

Time Period

1955-2009

Champions League Final KO Round

502

80

28

610

Europa League

Overall

0

0

536

536

Final KO Round

0

0

456

456

KO Round of last 16

0

0

80

80

Note: One observation is one game. Overall number of observations: 1,146. Final Knock-Out (KO) Round comprehends quarter- and semi-finals. We only consider games where the order of the home advantage is randomly assigned by the UEFA.

Table 2: UEFA regulation, passing the knock-out round and home advantage

\begin{tabular}{lrrlrr} 
UEFA Regulation & Observations & Frequency & Home Result & Observations & Frequency \\
\hline Goal difference & 929 & 0.81 & Home win & 611 & 0.53 \\
Away goals rule & 139 & 0.12 & Home draw & 300 & 0.26 \\
Supplementary time & 42 & 0.04 & Home defeat & 235 & 0.21 \\
Penalty kicks & 36 & 0.03 & & & \\
\hline
\end{tabular}

Note: There are several ways of passing the knock-out round as discussed in the appendix. This table summarizes the relative importance of each of them. The 'goal difference' regulation is the most important one. For a detailed description of the regulation see UEFA.com. 'Home Result' is defined as the result from the perspective of the home team, irrespective of whether it is the first or second game of the knock-out. One observation is one game. Overall number of observations: 1,146. We only consider games where the order of the home advantage is randomly assigned by the UEFA. 
Table 3: The absence of order effects

(1)

(2) (3)

(4)

(5)

(6)

Binary Dependent Variable: Win the knock-out $=1$; Lose $=0$

Second Home (SH)

$\begin{array}{cccccc}0.175 & 0.0873 & 0.0413 & 0.177 & 0.108 & -0.0288 \\ (0.167) & (0.296) & (0.300) & (0.190) & (0.203) & (0.315)\end{array}$

Round of last $16 * \mathrm{SH}$

$0.385 \quad 0.392$

(0.353) (0.348)

Quarter Final*SH

$0.123 \quad 0.172$

$(0.195) \quad(0.424)$

Semi Final*SH

$-0.0382-0.00463$

$(0.237) \quad(0.448)$

Champions League*SH

$0.466-0.00431$

0.419

$(0.413) \quad(0.168)$

$(0.556)$

Year Dummy

Yes

Yes

Constant

$\begin{array}{llllll}-0.0873 & -0.0873 & -0.0873 & -0.0873 & -0.0873 & -0.0873\end{array}$

$\begin{array}{lllllll}(0.0837) & (0.0837) & (0.0837) & (0.0837) & (0.0837) & (0.0837)\end{array}$

Observations

1,146

$1,134 \quad 1,134$

1,146

1,146

1,134

Note: Robust standard errors clustered by knock-out-ID in parentheses. The results do not change qualitatively if the probit regression model is used instead of the logit model. Year Dummy is a binary variable which indicates the year in which the knock-out is played. One observation is one game. We only consider games where the order of the home advantage is randomly assigned by the UEFA. 6 observations from Saison 1968 and 6 observations from Saison 2001 are dropped due to collinearity in specifications 2,3 and 6 . 
Figure 1: Results in the First Game (FG) and Second Game (SG)

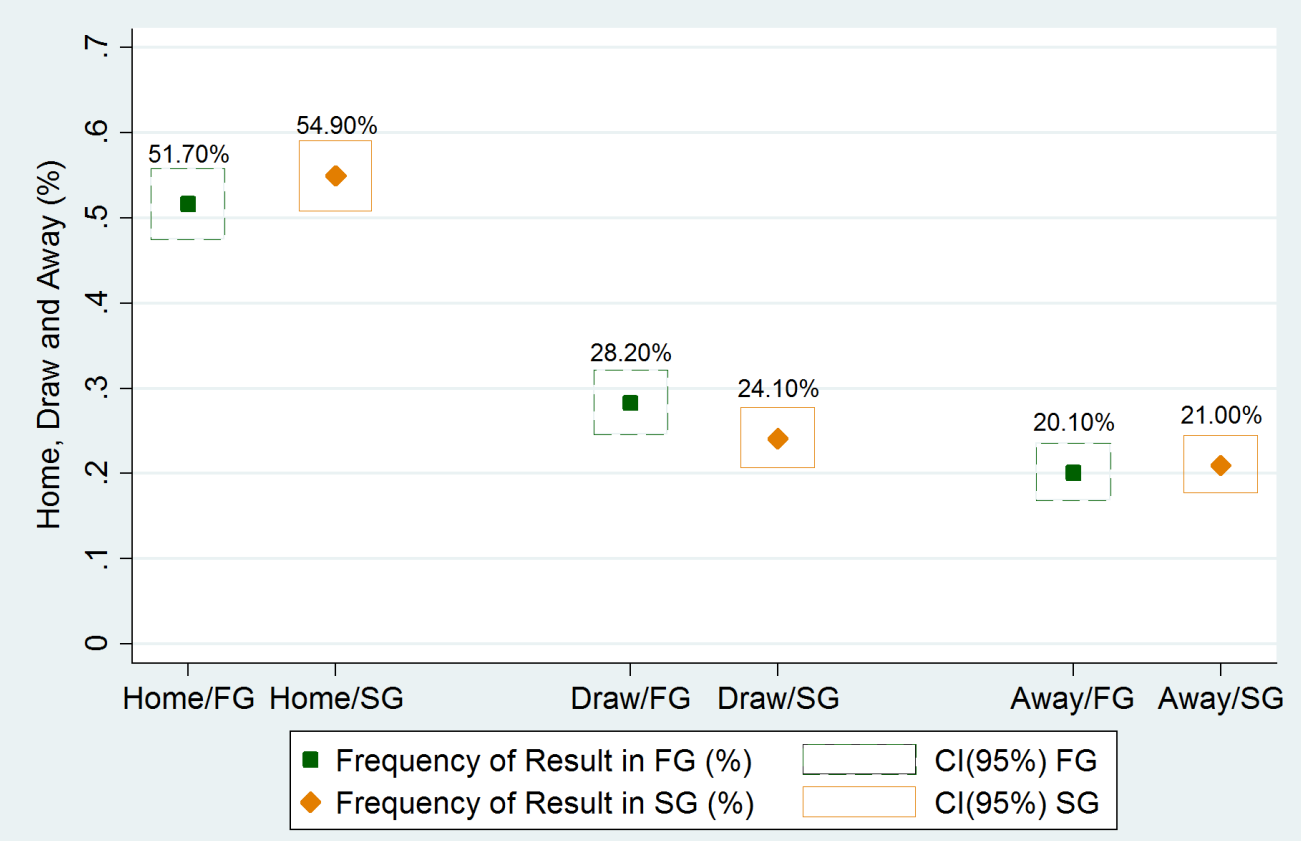

Note: Figure 1 shows the frequency of outcomes (home (win), draw, away (win)) in the first and second game of the knock-out. The square dot represents the percentage of each result, while the square around the dot represents the 95\% confidence interval (CI) around the mean. The square (diamond) represents the results in the first (second) game. FG (SG) refers to the first (second) game of the knock-out. The main result shown in Figure 1 is that the distribution of outcomes is statistically the same across games, a result confirmed by a non-parametric Kolmogorov-Smirnov where we cannot reject the null of equal distributions across games at a $p$-value of .89 . 
Figure 2: Sum of home and away goals by game

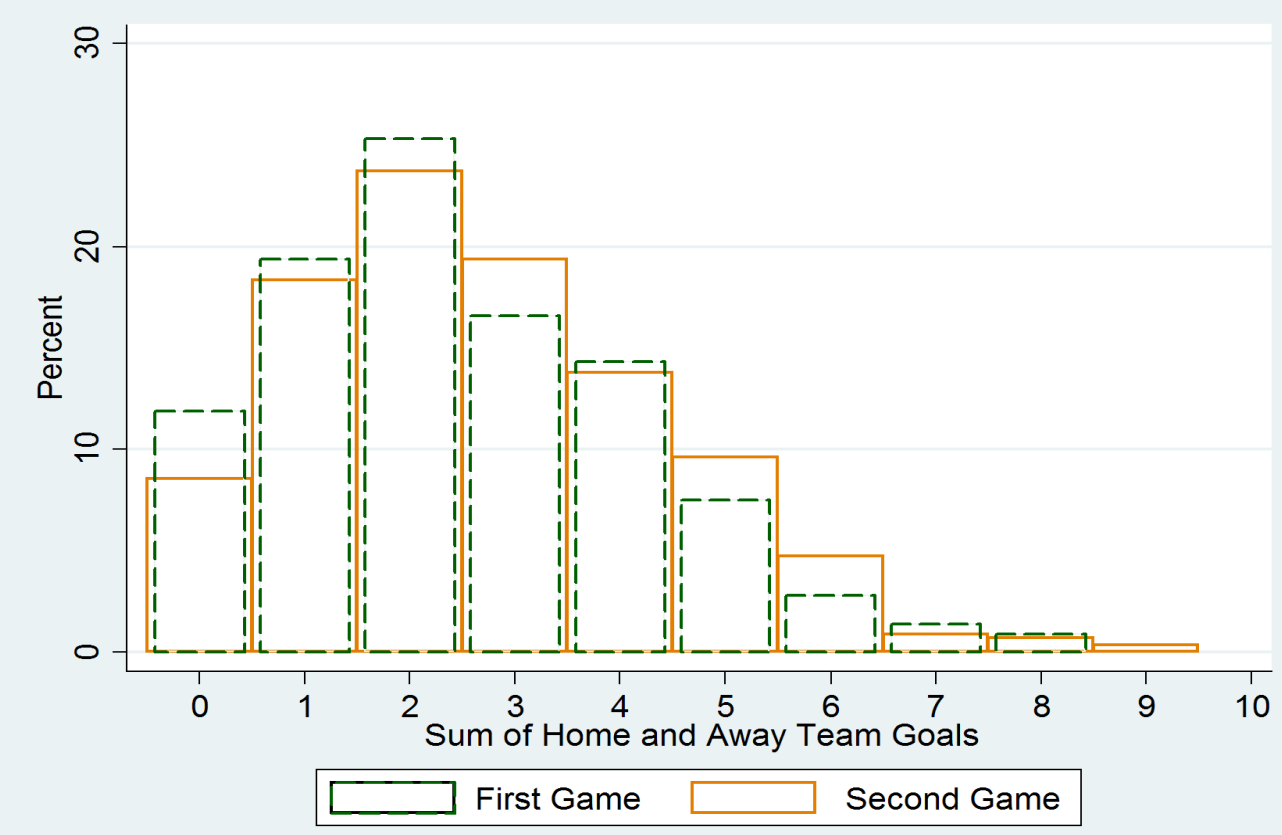

Note: Figure 2 shows the distribution of the sum of home and away team goals in the first and second game. The dotted (normal) bar represents the first (second) game. While the average number of goals in the second game is slightly higher than in the first game (2.7 vs. 2.48) a non-parametric KolmogorovSmirnov test cannot reject that the distributions are equal across games with a $p$-value of .24 . This shows that risk-taking measured by the distribution of the sum of goals is constant across games. 
Figure 3: Past performance and risk-taking

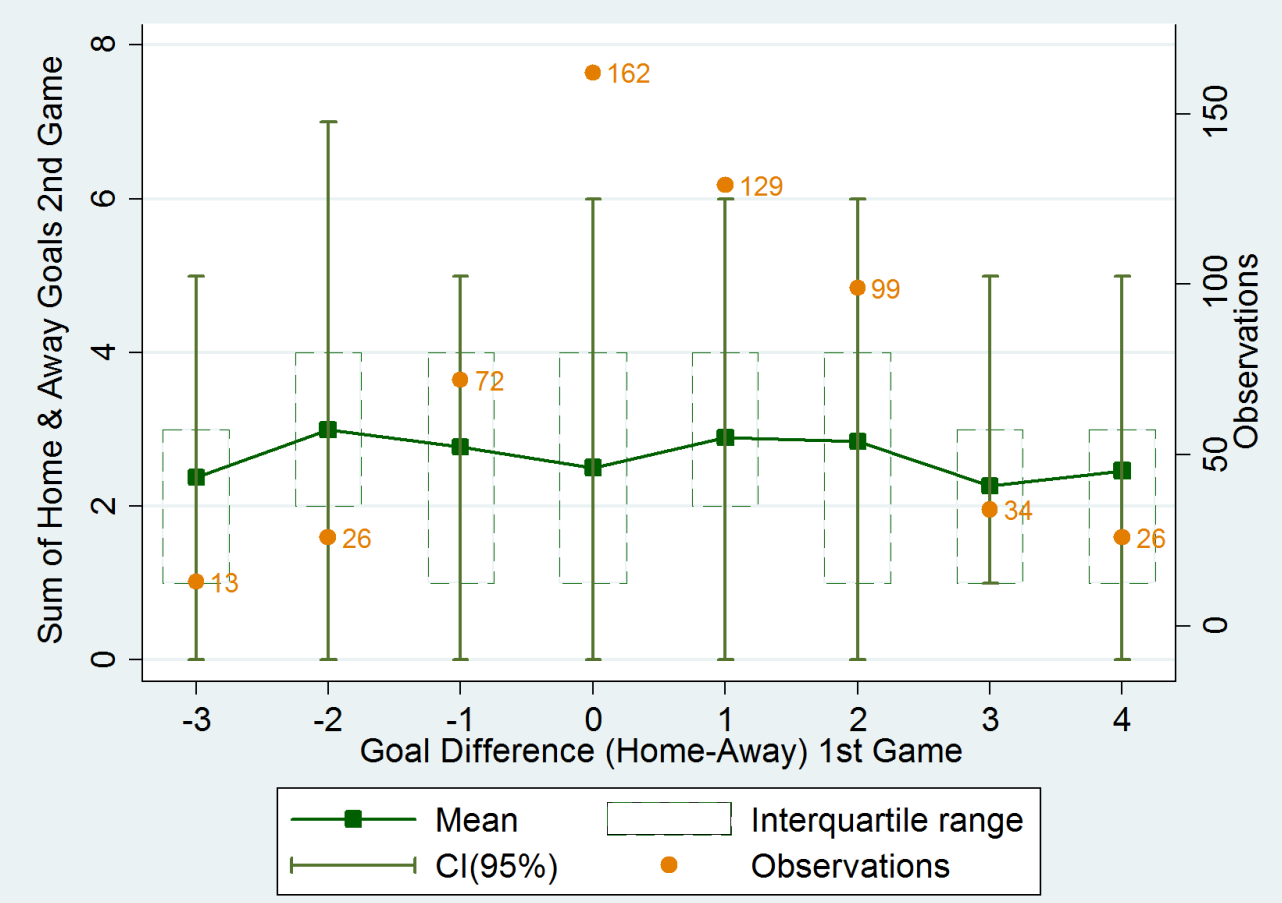

Note: Figure 3 shows the relation between the sum of home and away goals in the second game (left $y$-axis) and the goal difference (home-away) in the first game ( $x$-axis). In addition, the observations (knock-outs; on the right $y$-axis) to compute the mean, the interquartile range and the $95 \%$ confidence interval (CI) are reported. One observation is one knock-out round consisting of the first and second game. We restrict the sample to goal-differences with more than 10 observations. A total of 12 knockouts with goal-differences greater (lower) than $4(-3)$ are dropped. The main result of Figure 3 is that the sum of home and away goals in the second game is independent from the goal difference in the first game. If teams lagging behind increase risk-taking we would expect the sum of goals to increase with the absolute goal difference in the first game. The independence between these variables provides further evidence that teams do not change risk-taking as a response to past performance. 
Figure 4: Description of knock-out structure

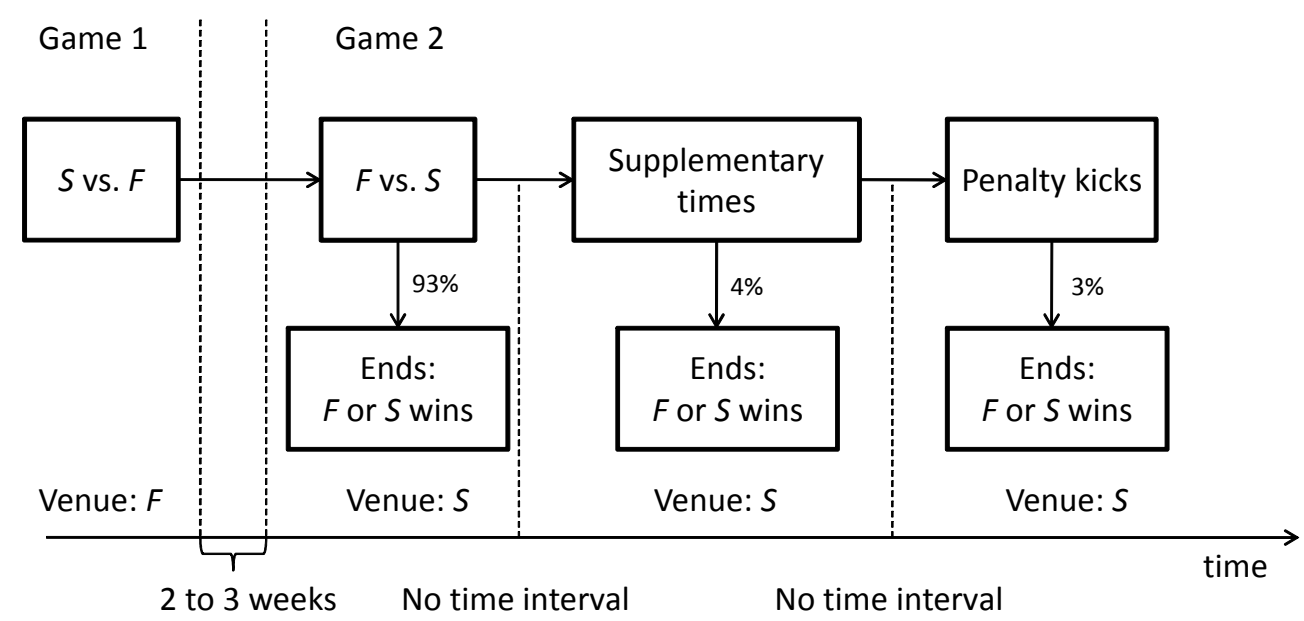

Note: $F(S)$ denotes the team playing the first (second) game at home. The arrows indicate the progress of the knock-out. $93 \%$ of the knock-outs end after the second game, $4 \%$ end after supplementary times and 3\% end after penalty kicks. 